\title{
Adaptation to visual and proprioceptive rearrangement: Origin of the differential effectiveness of active and passive movements
}

\author{
JAMES R. LACKNER \\ Brandeis University, Waltham, Massachusetts 02154
}

\begin{abstract}
In Experiment 1, subjects exposed to a discordance between the visual and "proprioceptive" locations of external targets were found to exhibit aftereffects when later pointing without sight of their hands at visual targets. Aftereffects uccur both when the discordance is introduced in the traditional fashion by displacing the visual locations of targets and when the proprioceptive locations of targets are displaced. These observations indicate that there is nothing unique about the visual rearrangement paradigm - the crucial factor determining whether adaptation will be elicited is the presence of a discordance in the positional information being conveyed over two different sensory modalities. In a second experiment, the effectiveness of active and passive movements in eliciting adaptation was studied using an experimental paradigm in which subjects were exposed to a systematic discordance between the visual and proprioceptive locations of external targets without ever being permitted sight of their hands; a superiority of active movements was observed, just as is usually found in visual rearrangement experiments in which sight of the hand is permitted. Evidence is presented that the failure of passive movements to elicit adaptation is related to a deterioration in accuracy of position sense information during passive limb movement.
\end{abstract}

Considerable effort has been devoted to determining the conditions under which adaptation to visual rearrangement occurs in the adult, because it has been suggested (Held \& Bossom, 1961; Held \& Hein, 1958) that the processes mediating restoration of accurate sensory-motor coordination are similar to those involved in the initial acquisition of coordinated movement by the neonate. Moreover, an understanding of the sensory factors that influence adaptation to visual rearrangement and the specific adaptive changes that occur to eliminate the disruptive consequences of sensory rearrangement should furnish valuable clues about the hierarchical organization of sensory-motor control.

Some progress toward specifying these factors has been achieved using an experimental paradigm that permits the independent manipulation of the apparent visual and apparent proprioceptive loci of external targets (Lackner, 1973a, 1974a). Experiments using this technique have demonstrated that if subjects are not permitted visual feedback about the accuracy of their pointing movements during exposure to visual rearrangement, then compensatory changes in sensory-motor coordination do not occur unless a discordance exists between the visually and proprioceptively specified directions of the visual

Support was provided by the Rosenstiel Biomedical Sciences Foundation, the Spencer Foundation, and NASA Grant 22-009-308. targets (Lackner, 1974a).$^{1}$ In these experiments, subjects were not permitted sight of their hands at any time and adaptation was elicited solely on the basis of a conflict between sensory inputs arriving through two modalities, vision and proprioception.

Related experiments-using exposure conditions in which sight of the hand is permitted-in which visual, efferent, and proprioceptive information about hand position have been systematically varied also emphasize the importance of visual-proprioceptive discordances in eliciting adaptation (Mather \& Lackner, 1975, 1976; Rock, 1966; Wallach, 1968).

The first experiment to be described here investigates whether the direction of a visual-proprioceptive discordance is critical; that is, whether the existence of a discordance between the visual and proprioceptive locations of external targets will lead to changes in pointing movements to these targets even if the targets' visual images are not displaced. This question warrants consideration because ordinarily vision is thought to dominate proprioception; when the two are in conflict, the visual localization generally predominates (Rock \& Harris, 1967). Thus, for example, when one looks at his arm through prism spectacles, he feels it where he sees it. Harris $(1963,1965)$ has demonstrated that, over time, a change in apparent position of the limb persists regardless whether the eyes are open or closed. This change is such that the "felt position" of the limb is displaced in the direction of the visual 
deviation (e.g., for a leftward visual displacement, the limb will be perceived as being aligned with the mid-saggital body plane when it is actually to the right of that plane); the change is adaptive because it automatically restores accurate visuomotor coordination with the exposed limb.

Such postural compensations are a regular aspect of adaptation to visual rearrangement when sight of the hand is permitted. To make sure that the same kinds of compensatory changes are evoked in the exposure conditions of the present experiments-in which visual-proprioceptive discordances are created but sight of the hand is withheld-it was essential to include a measure of "pointing straight ahead," which would reveal any changes in position sense of the limb used during exposure.

\section{EXPERIMENT 1}

The experimental design involved measuring and comparing the accuracy with which subjects pointed to visual targets before and after an exposure period in which they received systematic proprioceptive misinformation about the locations of visual targets. The results indicate, in conjunction with previous observations, that whenever there is a discrepancy between the visual and proprioceptive loci of external targets there is a shift in pointing movements to these visual targets and to the straightahead of the body.

\section{Method}

Apparatus. The experimental apparatus has been described in detail elsewhere (Lackner, 1973a). It permits the creation of sensory discordances between the visual and proprioceptive directions of external targets. The subject sits at one end of the apparatus and is permitted sight of four visual targets (metal pins $3 \mathrm{in.}$ high); an opaque shelf extending forward horizontally from the level of his chin prevents him from seeing his hand at any time during the experiment.

The apparatus has provisions for recording a subject's accuracy in pointing to visual targets during pre- and postexposure measurement conditions. A curved surface, faced with conducting Teledeltos paper, can be positioned vertically below the visual targets; a voltage gradient exists across this surface, and when the subject touches the surface with a stylus, the exact horizontal location of contrast is indicated by the reading on a digital voltmeter ${ }^{2}$ and permanently recorded by a digital printer. The apparatus also has, by means of metal pins that can be raised into position below the level of the target pins, provisions for giving the subject (either accurate or inaccurate) proprioceptive feedback about the location of the visual targets. These lower pins can be placed in any desired angular relation to the top pins, and their position can be changed during the experiment without the subject's knowledge.

Subjects. Sixteen male Brandeis undergraduates were paid for their voluntary participation; all had normal vision without glasses or with contact lens correction.

Procedure. The experiment was divided into preexposure, exposure, and postexposure periods. In the preexposure period, the subject was required to reach forward with a metal stylus and touch horizontally just below the apparent locations of the visual targets; the subject pointed as if the visual target pin continued downward below his line of sight. After each pointing response, the subject returned his hand to his lap before marking the location of the next target. The subject pointed in turn, left to right, at visual targets located $30^{\circ}$ left, $10^{\circ}$ left, $10^{\circ}$ right, and $30^{\circ}$ right of his midline. He then pointed to the straightahead while keeping his eyes closed. In pointing to the "straightahead," the subject was attempting to place the stylus in the projection of the mid-saggital plane of his body at the distance of the recording surface. The subject repeated the entire sequence until he had completed a total of 100 responses. The preexposure period was preceded by 16 responses to familiarize the subject with the demands of the task.

For the exposure condition, the recording surface was removed and the bottom proprioceptive-feedback pins were raised into position. The subject was then required to look at each visual target in turn while reaching forward just below the visual target and moving his hand until he made contact with the metal feedback pin corresponding to the target pin; while doing this, the subject held the marking stylus in his hand so that his movements would be similar throughout all conditions of the experiment. The subject pointed in this fashion to the targets, left to right, returning his hand to his lap between movements; he did not point to the straightahead during the exposure period. In the exposure condition, as well as in the pre- and postexposure conditions, the subject's movements were paced to the beat of a metronome, one movement each $3 \mathrm{sec}$.

Initially, the proprioceptive feedback pins formed downward extensions of the visual target pins, but after every cycle of pointing to the four targets the bottom pins were displaced $1 / 4^{\circ}$ in relation to the visual target pins. Consequently, after 40 complete cycles there was a $10^{\circ}$ offset between the visual pins and the proprioceptive target pins. With the exception that no practice trials were involved, the postexposure condition was identical to the preexposure condition. Eight of the 16 subjects were exposed to a visual-proprioceptive discordance in which the proprioceptive locations of the targets were to the left of the visual locations and five were exposed to the opposite pattern of displacement.

\section{RESULTS}

The experimental findings are summarized in Table 1. Responses were averaged across subjects and target positions separately for the two directions of rearrangement. The difference between the preand postexposure averages was used as a measure of adaptation. For both directions of proprioceptive rearrangement, significant differences were observed between pre- and postexposure scores, both for pointing at visual targets $(p<.02$ for leftward proprioceptive displacements and $\mathrm{p}<.01$ for rightward displacements, two-tailed t test) and for pointing straightahead ( $p<.02$; two-tailed t test). If the proprioceptive loci of the visual targets had been displaced leftward in relation to the visual loci during the exposure conditions, then in the postexposure period the subject always erred to the left when pointing at visual targets and when pointing to the straightahead; the opposite pattern was elicited by rightward proprioceptive displacements.

Each subject, after completing the experiment and withdrawing from the apparatus, continued to make errors when reaching quickly for objects, e.g., when trying to touch his nose or the experimenter's upheld index finger. 
Table 1

Mean Aftereffects in Degrees for Pointing at Visual Targets and Pointing to the Median Plane of the Body After Exposure to Proprioceptive Rearrangement

Proprioceptive Deplacement (Degrees)

\begin{tabular}{clcc}
$\begin{array}{c}\text { Pointing } \\
\text { Objective }\end{array}$ & & $\begin{array}{c}\text { 10-Deg } \\
\text { Left }\end{array}$ & $\begin{array}{c}10-\text { Deg } \\
\text { Right }\end{array}$ \\
\hline \multirow{2}{*}{ Visual Targets } & Mean & $+3.9^{*}$ & $+5.2 \dagger$ \\
& SD & 1.3 & 1.4 \\
Straightahead & Mean & $+4.2^{*}$ & $+4.9^{*}$ \\
& SD & 1.3 & 1.6 \\
\hline
\end{tabular}

Note-The plus sign indicates that the direction of the aftereffect corresponds to the direction of the proprioceptive displacement.

* Significantly different from zero $(p<.02)$

Significantly different from zero $(p<.01)$

\section{Discussion}

It is clear from the experimental observations that there is nothing unique about the visual rearrangement paradigm; exposure to proprioceptive rearrangement is equally effective in eliciting modifications in sensory-motor coordination. The critical factor determining whether adjustments will occur is the presence or absence of a discordance between the visual locations of body parts (or external objects) and proprioceptive locations (Lackner, 1974a; Mather \& Lackner, 1975, 1976); this discordance need not be consciously recognized by the subject in order for adaptation to occur. Furthermore, when a discrepancy exists between the spatial information about limb position being supplied by vision and touch, there is a change in the centrally interpreted position of that limb, as indicated by the change in pointing to the straightahead. It should be noted, however, that changes in apparent head position may occur as well in such exposure situations-especially when the subject's head and trunk are not both restrained from moving (Lackner, 1973b, c, 1974b).

A reinterpretation of arm position during visual rearrangement automatically restores the accuracy of pointing movements made to external visual targets because the shift in apparent arm position compensates for the displaced visual locations of the external targets. However, the adapted subject will err when pointing to the straightahead or positions on his own body; for instance, if he attempts to touch his nose or to set his hands to a particular judged separation while his eyes are closed, he will err by an amount and in a direction determined by the change in apparent position of his adapted arm (Harris, 1963, 1965). Accordingly, the change in apparent position of the arm seen during exposure to visual rearrangement is adaptive for pointing movements made to external visual targets and maladaptive for pointing movements made to the body itself. Proprioceptive rearrangement also leads to a reinterpretation of the apparent position of the exposed arm with respect to the rest of the body and therefore to errors in body-relative orientational movements; however, it creates errors in pointing responses to visual targets as well. The change in position sense during exposure to proprioceptive rearrangement is thus maladaptive both with respect to pointing movements made to visual targets and to the rest of the body.

\section{EXPERIMENT 2}

It may be asked whether the foregoing observations have general significance for experimental situations in which subjects are permitted sight of their hands during exposure to visual rearrangement. A persistent finding in such experiments is the superiority of active over passive body movements in producing compensatory changes in eye-hand coordination (Held, 1961, 1965; Held \& Hein, 1958; Wooster, 1923). The apparently overwhelming superiority of active movements in producing adaptation spurred the formulation of models in which the basis for adaptation was attributed to a mechanism by which corollary discharge information about ongoing voluntary movements was correlated with reafferent visual feedback generated by those movements (Held, 1961, 1968). Adaptation was thought not to occur during passive movements because of the absence of corollary discharge signals and accordingly the lack of a basis for correlating efferent and afferent information about limb position.

Although these models have been shown both on logical and experimental grounds to be of limited value (Lackner, 1974a; Mather \& Lackner, 1975, 1976), the superiority of active to passive body movements in producing adaptation nevertheless requires explanation. One reason for such a difference is suggested by the demonstration of Paillard and Brouchon $(1968,1974)$ that position sense is considerably more accurate for an actively moved, or actively positioned, limb than for a passively moved limb. This differential accuracy of limb-position specification relates to the availability of proprioceptive information from muscle spindles during voluntary, but not passive, movements. As Paillard and Brouchon (1968) hypothesized, and Goodwin, McCloskey, and Matthews (1972a, b) later proved, muscle spindle information contributes to the position sense of our limbs primarily during voluntary movements. It seemed possible therefore that in the case of an arm passively moved during exposure to visual rearrangement, the discordance between its seen position and proprioceptively specified position with respect to the body might be less accurately registered than when the limb was actively moved. If so, this difference would account 
for the greater efficiency of active movements in eliciting adaptation. The present experiment using a variation on the exposure condition of Experiment 1 was undertaken to evaluate this possibility. It involved creating a discordance between the visually and proprioceptively specified directions of external targets by displacing the visual locations of the targets. A visual, rather than proprioceptive, displacement was used so that the exposure period would mimic the sensory conditions characteristic of visual rearrangement experiments using traditional exposure conditions.

\section{Method}

Apparatus. The apparatus used in Experiment 1 was modified to provide for active and passive movements of the arm of matched extent and velocity. An armrest supporting the entire length of the subject's arm and permitting horizontal, but not vertical, movements of the subject's arm was added to the apparatus. When strapped in the support, the subject's arm is in the neutral position (i.e., midway between pronation and supination), the position normally assumed when pointing to objects. The arm can be moved at the shoulder joint but not at the elbow joint, the fingers and hand are free to move.

Subjects. Twelve right-handed Brandeis undergraduates participated as paid volunteers. All were male because of the requirement that their right arms had to be long enough to comfortably reach $40^{\circ}$ to the left or right of the body midline at a distance of 24 in.; most Brandeis females have arms shorter than the required length.

Procedure. The experiment was divided into three stages: preexposure, exposure, and postexposure. During the pre- and postexposure trials, the subject pointed in turn to the apparent loci of four visual targets, located at $30^{\circ}$ left, $10^{\circ}$ left, $10^{\circ}$ right, and $30^{\circ}$ right in relation to his midline. The subject's right arm was in the support and was always moved voluntarily by him until he thought his hand was directly beneath a target pin. $\mathrm{He}$ then marked the apparent position of the target with a stylus, which he held in his fingers, by pushing the stylus forward against the marking surface. The subject pointed to each target in turn moving from left to right; he repeated this sequence 25 times. Before the preexposure, but not the postexposure, condition, the subject was allowed five practice sequences to permit adjustment to the demands of the task. During preand postexposure trials, the subject paced his movements to a metronome, and each $2 \mathrm{sec}$ he had to point to one of the targets.

Every subject participated in two experiments separated by at least 3 days [this interval was chosen because adaptive modifications engendered by exposure to visual arrangement can persist longer than $24 \mathrm{~h}$ (Lackner \& Lobovits, 1976)]. On one day, a subject had an active-exposure condition, on the other, a passive-exposure condition. The order of active and passive exposure was balanced across subjects. During active-exposure trials, the subject moved his arm and the support from left to right and back while fixating, in turn, the visual targets as his fingers encountered the corresponding bottom pegs. The subject let his fingers touch and brush past the bottom pegs allowing his hand and arm to move on without interruption. Immediately after passing a peg, the subject returned his fingers and hand to the neutral position in preparation for the next peg. The subject moved his arm in time to the beat of a metronome, completing one left-right (or right-left) sweep each $4 \mathrm{sec}$. For the passive exposure condition, the subject was instructed to let his arm muscles remain flaccid, and the experimenter, by means of a handle attached to the armrest, moved the subject's arm back and forth, in time with the metronome beat as in the active condition. While his arm was being moved in the passive condition, the subject, while fixating the appropriate visual targets, let
Table 2

Mean Aftereffects in Degrees Elicited by Active and Passive Movements During Exposure to 10-Deg Leftward and 10-Deg Rightward Visual Displacements

\begin{tabular}{clcc} 
& & \multicolumn{2}{c}{ Visual Displacement } \\
\cline { 3 - 4 } $\begin{array}{c}\text { Movement } \\
\text { Type }\end{array}$ & & $\begin{array}{c}\text { 10-Deg } \\
\text { Left }\end{array}$ & $\begin{array}{c}\text { 10-Deg } \\
\text { Right }\end{array}$ \\
\hline \multirow{2}{*}{ Active } & Mean & $+3.3^{*}$ & $+3.8^{*}$ \\
& SD & 1.2 & 1.3 \\
Passive & Mean & $+0.6 \dagger$ & $+1.3 \dagger$ \\
& SD & 1.2 & 1.4 \\
\hline
\end{tabular}

Note-All aftereffects were in a compensatory direction. *Significantly different from zero $(p<.025)$ $\dagger$ Not significantly different from zero $(p<.05)$

his fingers touch and brush past the feedback pins, and then, as in the active exposure condition, returned them to the neutral position.

In the exposure trials, six subjects were given $10^{\circ}$ leftward visual displacements and six $10^{\circ}$ rightward displacements. A given subject always received the same direction of visual displacements during his active- and passive-exposure sessions. The difference between a subject's pre- and postexposure scores served as an index of the amount of adaptation generated by the exposure condition.

\section{Results}

Table 2 summarizes the experimental results. Preand postexposure scores were averaged across target positions and subjects separately for the two displacement directions. For both rightward and leftward visual rearrangements, the active exposure conditions led to significant adaptation, although somewhat greater adaptation occurred for rightward visual displacement. The aftereffects generated by the passive exposure conditions failed to reach significance for either the leftward $(p>.05)$ or rightward $(p>.05)$ visual displacements.

\section{Discussion}

It is known from the work of Paillard and Brouchon $(1968,1974)$ and earlier investigators (reviewed by Granit, 1972) that during and following passive movements of the arm, subjects are less accurate in their judgment of arm position than when they are permitted voluntary control. This loss of fine resolution results apparently from the absence of utilizable muscle spindle information during passive limb movements (cf. Goodwin, McCloskey, \& Matthews, 1972a, b; McCloskey, 1974; McCloskey, Ebeling, \& Goodwin, 1974). These observations, in conjunction with a specification of the sensory conditions that must prevail before adaptation to visual rearrangement can occur, form a basis for understanding the origin of active-passive differences in sensory-rearrangement experiments.

Earlier experiments utilizing exposure conditions that deny a subject visual information about the size and direction of misreaching errors demon- 
strated that adaptation to visual rearrangement occurs only when there is a discordance between the visually and proprioceptively specified directions of the hand (Mather \& Lackner, 1975, 1976) or external targets (Lackner, 1974a) and that corollary discharge signals contribute little if at all to the adaptive process. Here we have found that, under comparable experimental conditions, passive movements of the arm fail to elicit significant adaptation. Consequently, it appears that when passive movements are made during exposure to visual rearrangement there is a less effective specification of the discordance between the visual and proprioceptive locations of targets than when active movements are executed. One aspect of the superiority of active over passive movements in producing adaptation to visual rearrangement thus relates to the greater accuracy of position sense information about voluntarily moved limbs, a greater accuracy derived in part from the contribution of muscle afferent signals.

It should be noted, however, that the superiority of active movements is but a relative one. Passive movements elicit significant adaptation in exposure conditions in which the frequency and amplitude characteristics of passive limb displacement and the total duration of exposure are chosen so as to enhance positional information about limb orientation (cf. Lackner, 1976; Mather \& Lackner, 1976).

\section{REFERENCES}

Bauer, J. A., Woods, G. D.. \& Held, R. A device for rapid recording of positioning responses in two dimensions. Behavior Research Methods \& Instrumentation, 1969, 1, 157-159.

Goodwin, G. M., McCloskey, D. I., \& Matrhews, P. B. C. Proprioceptive illusions induced by muscle vibration: Contribution to perception by muscle spindles? Science, 1972, 175, 1382-1384. (a)

Goodwin, G. M., McCloskey, D. I., \& Matrhews, P. B. C. The contribution of muscle afferents to kinesthesia shown by vibration induced illusions of movement and by the effects of paralysing joint efferents. Brain, 1972, 95, 705-748. (b)

Granit, R. Constant errors in the execution and appreciation of movement. Brain, 1972, 95, 649-660.

HARRIS, C. Adaptation to displaced vision: Visual, motor, or proprioceptive change? Science, 1963, 140, 812-813.

Harris, C. Perceptual adaptation to inverted. reversed, and displaced vision. Psychological Review, 1965, 72, 419-444.

Held, R. Exposure history as a factor in maintaining stability of perception and coordination. Journal of Nervous and Mental Diseases, 1961, 32, 26-32.

HELD, R. Plasticity in sensory motor systems. Scientific American, 1965. 213, 84-94.

HELD, R. Action contingent development of vision in neonatal animals. In D. Kimble (Ed.), Experience and capacity. New York: New York Academy of Sciences, 1968.

Held, R., \& Bossom, J. Neonatal deprivation and adult rearrangement. Joumal of Comparative and Physiological Psychology, 1961, 54, 33-37.
Held, R., \& Hein, A. Adaptation to disarranged hand eye coordination contingent upon re-afferent stimulation. Perceptual and Motor Skills, 1958, 8, 87-90.

Howard. I., \& Templeton, W. Human spatial orientation. New York: Wiley, 1966.

LACKNER, J. A device for investigating adaptation to sensory rearrangement. Journal of Psychology, 1973, 85, 137-141. (a)

LACKNER, J. Visual rearrangement affects auditory localization. Neumpsychologia, 1973, 11, 29-32. (b)

LACKNER, J. The role of posture in adaptation to visual rearrangement. Neuropsychologia, 1973, 11, 33-44. (c)

LACKNER, J. Adaptation to displaced vision: Role of proprioception. Perceptual and Motor Skills, 1974. 38. 1251-1256. (a)

LACKNER. J. Influence of visual motion and visual rearrangement on sound localization. Neuropsychologia, 1974, 12, 291-293. (b)

LACKNER, J. Influence of abnormal postural and sensory conditions on human sensorimotor localization. Environmental Biology and Medicine, 1976, 2. 139-177.

LACKNER, J., \& LoBovits, D. Adaptation to displaced vision: Evidence for prolonged aftereffects. Quarterly Journal of Experimental Psychology, in press, 1976.

MATHER, J., \& LACKNER, J. Adaptation to visual rearrangement elicited by tonic vibration reflexes. Experimental Brain Research. 1975. 24, 103.105.

MATher, J., \& LACKNER, J. Relative contributions of proprioceptive, corollary-discharge, and visual information during adaptation to visual rearrangement. Quarterly Journal of Experimental Psychology, in press. 1976.

MCCloskeY, D. I. Muscular and cutaneous mechanisms in the estimation of the weight of grasped objects. Neuropsychologia. $1974,12,513-520$.

McCloskey, D. 1., Ebeling, P., \& Goodwin, G. Estimation of weights and tension and apparent involvement of a "sense of effort." Experimental Neurology, 1974, 42, 220-232.

Paillard, J., \& Brouchon, M. Active and passive movements in the calibration of position sense. In S. Freedman (Ed.), The neuropsychology of spatially oriented behavior. Homewood, Ill: Dorsey, 1968. Pp. 37-55.

Paillard, J.. \& Brouchon, M. A proprioceptive contribution to the spatial encoding of position cues for ballistic movements. Brain Research, 1974, 71, 273-284.

Rock, 1. The nature of perceptual adaption. New York: Basic Books, 1966.

Rock, I., \& Harris, C. Vision and touch. Scientific American, 1967. 216, 96-104.

WALLACH, H. Information discrepancy as a basis of perceptual adaptation. In S. Freedman (Ed.). The neuropsychology of spatially oriented behavior. Homewood, Ill: Dorsey, 1968. Pp. 209.229 .

Wooster. M. Certain factors in the development of a new spatial coordination. Psychological Monographs, 1923, 32(4. Whole No. 146).

\section{NOTES}

1. Wallach (1968) argued forcefully several years ago that such discordances might very well be necessary to elicit adaptive changes. Similar views were expressed by Howard and Templeton (1966).

2. This technique for recording positioning responses was introduced by Bauer, Woods, and Held (1969).

(Received for publication August 11, 1976; revision accepted October $22,1976$. 